\title{
Forming Successful Unconventional Collaborations
}

\author{
Annie Sobel
}

Assistant to the Provost and the Vice President, University of Missouri

$\mathrm{F}$

orming and sustaining unconventional collaborations is an opportunity to advance knowledge in unanticipated and sometimes surprising ways. Working at the fringes of interdisciplinary work may be exciting and deeply rewarding. The exploration of relationships, ideas, cultures, and the range of scientific disciplines define the edges of innovation and entrepreneurship. This brief paper will describe some emerging unconventional opportunities for collaboration in the life sciences.

The formation of successful unconventional collaborations is often catalyzed through compelling need and a greater understanding of nature, her underlying processes and their complex interactions. The path to collaboration emerges through "self-assembly" of the critical disciplines, and often stresses the "liminal", previously unconsidered areas. For example, in the field of biophysics, natural collaborators have self-organized through a growing understanding of mechanisms of cellular communication, expression of proteins and metabolomes. The dynamic interrelationships that enable cell signaling and trafficking has emerged as an important discipline in the understanding of living systems.

An important international example of the value of collaborations is the Cooperative Threat Reduction (CTR) program. While intended to facilitate transition of science and technology research from nefarious to legitimate purposes specifically in the fields of

biological and chemical engineering, many contributions to global societies have emerged from this program. International cooperation was strengthened, new interdisciplinary research and entrepreneurship opportunities ensued, and previously unrecognized science talent was recognized and funded. In addition, new opportunities for student research and interdisciplinary teaching arose, and scientists began applying resources to a new set of challenges that would play significant roles in advancing medical therapeutics, diagnostics and disease prevention.

Navigating and fertilizing the landscape to promote successful collaboration is often challenging. First, the case for grass roots collaboration is often self-evident and an important initiator for the work, but requires continued nurturing through resources and institutional support to remain sustainability. 
Collaboration may sometimes be described as schizophrenic, since the optimal pathway to success often benefits from multiple parallel, and sometime conflicting, initiatives. For example, in the life sciences area, successful programs evolve that address system, cellular and sub-cellular level questions and translational opportunities. The translational opportunities may emerge as entrepreneurial and/or sustainable joint scientific ventures. Success may be defined on many levels, from research publication, to scientific truth, to new opportunities for commercialization, to launching students on a path to a lifetime of scientific discovery.

Collaborations may evolve in a number of ways. The most commonly observed collaborations demonstrate convergence on a challenging set of problems and issues that naturally may be characterized as multi-dimensional or inter-disciplinary; unconventional collaborations may evolve in this way. Other collaborations tend to thrive on fractal formation, with many branch points emerging from the research and entirely new areas for scientific pursuit emerging. In addition, complementarities of purpose, process, and function may emerge from collaboration. Complementarities may be seen in bio-diverse systems and an understanding of how environmental stressors may push evolutionary and adaptation mechanisms.

Alternatively, collaborations may evolve toward divergence; specifically, as understanding of the problem or set of problems evolves, the manifest differences and incongruities become increasingly obvious. Most collaborations adapt to the pressures, both internal and external, that force them to either flourish (successful) or self-extinguish (unsuccessful). Clearly, there are a number of human and cultural factors that drive this process, however, the over-riding factor tends to be the compelling nature of the problemopportunity.

Unconventional collaborations may be catalyzed by emergencies. For example, climate change and planetary impact has facilitated the cooperation of green chemistry researchers, atmospheric modelers and medical researchers. The increasing prevalence of toxic environmental substances, global urbanization, and overabundant biomass in population dense neighborhoods creates a global melting pot, sort of a genetic soup for transmission and co-evolution of disease.

The significance of furthering our knowledge of an inter-species global health corridor and "collaboratory" of scientists worldwide is gaining traction. The biological sciences community has long understood the importance of tracking avian flight paths, however, only recently has a greater appreciation of opportunities for sentinel disease surveillance employing birds become popularized. Global spread and evolving pathogenicity, are enabled by environmental factors such highly efficient airborne routes of transmission of West Nile Virus, SARS, Avian and Swine influenza. Theses system-level observations emphasize the need for interdisciplinary, adaptive collaboration. Pathogen resistance to preventive 
medicine strategies (i.e., anti-viral cellular, and sub cellular-levels of agents) and the rapid, subtle mutations zoonotic diseases, coupled to the and genetic shifts underscore the complex interactions of humans, animals complex system dynamics and are just a and plants that express these diseases, few compelling examples of interactions renews the scientific community's between global communities of animals, enthusiasm for collaborations in global humans, and environment.

The expanding body of knowledge and understanding at the system, health, comparative and translational medicine for generations to come. 